\title{
Sequential disruption of ALV host receptor genes reveals no sharing of receptors between ALV subgroups A, B, and J
}

\author{
Hong Jo Lee ${ }^{1 \dagger}$, Kyung Je Park ${ }^{1 \dagger}$, Kyung Youn Lee ${ }^{1}$, Yongxiu Yao ${ }^{2}$, Venugopal Nair ${ }^{2}$ and Jae Yong Han ${ }^{1 *}$ (D
}

\begin{abstract}
Background: Previously, we showed that targeted disruption of viral receptor genes in avian leukosis virus (ALV) subgroups using clustered regularly interspaced short palindromic repeats (CRISPR)/CRISPR-associated protein 9 (Cas9))-based genome editing confers resistance to ALV subgroups B and J. Here, we used the same strategy to target the receptor expressed by ALV subgroup A (TVA) and generate chicken cells resistant to infection by this virus.

Results: CRISPR/Cas9-based disruption of exon 2 within the tva gene of DF-1 fibroblasts conferred resistance to infection by ALV subgroup A regardless of whether frameshift mutations were introduced during editing. Conversely, overexpression of the wild-type TVA receptor (wtTVA) by tva-modified DF-1 clones restored susceptibility to ALV subgroup A. The results confirm that exon 2, which contains the low-density lipoprotein receptor class A domain of TVA, is critical for virus entry. Furthermore, we sequentially modified DF-1 cells by editing the $t v a, t v b$, and $\mathrm{Na}^{+} / \mathrm{H}^{+}$ exchange 1 (chNHE1) genes, which are the specific receptors for ALV subgroups A, B, and J, respectively.

Conclusions: Simultaneous editing of multiple receptors to block infection by different subgroups of ALV confirmed that ALV subgroups A, B, and J do not share host receptors. This strategy could be used to generate cells resistant to multiple viral pathogens that use distinct receptors for cell entry.
\end{abstract}

Keywords: Avian leukosis virus, CRISPR/Cas9, Genome editing, Host receptor, TVA

\section{Introduction}

Avian leukosis viruses (ALV) are pathogenic avian retroviruses that cause major neoplastic diseases in many poultry flocks, resulting in significant economic losses to the global poultry industry [1]. While a number of poultry breeding companies have been successful at eradicated ALV infections from flocks, continued spread of ALV in some Asian countries remains an issue for the poultry market $[2-5]$.

ALVs are divided into different subgroups based primarily on the sequence of the envelop glycoprotein, which is the major determinant of the receptor interactions required for virus entry, host range, and virus

\footnotetext{
* Correspondence: jaehan@snu.ac.kr

${ }^{+}$Hong Jo Lee and Kyung Je Park contributed equally to this work.

${ }^{1}$ Department of Agricultural Biotechnology, College of Agriculture and Life

Sciences, and Research Institute of Agriculture and Life Sciences, Seoul

National University, Seoul 08826, Korea

Full list of author information is available at the end of the article
}

neutralization [6]. Each ALV subgroup targets specific host cell receptors to interact with the envelop glycoproteins; thus, viral entry into cells depends largely on dominant expression of a particular host receptor. ALV subgroup A enters host cells via the TVA receptor, which is a low-density lipoprotein receptor (LDLR) [7]. ALV subgroups $B, D$, and E enter cells via the TVB receptor, a tumor necrosis factor receptor (TNFR)-related protein [8-10]. ALV subgroup C uses the TVC receptor, which is related to the mammalian butyrophilins [11], and ALV subgroup $\mathrm{J}$ uses chicken $\mathrm{Na}^{+} / \mathrm{H}^{+}$exchanger type 1 (chNHE1) [12].

Genetic variations in host receptor genes determine the susceptibility of chickens to infection by different ALV subgroups. A single base pair mutation resulting in a cysteine to tryptophan mutation or a four base pair insertion into exon 1 of the tva gene confers resistence to ALV subgroup A viruses [13]. In addition, intronic deletions within the tva receptor gene that disrupt mRNA 
splicing confer resistance to ALV subgroup A viruses $[14,15]$. In addition, a naturally occurring premature stop codon or a single amino acid substitution within the $t v b$ gene leads to a marked reduction in susceptibility to ALV subgroups B, D, and E [16, 17]. Furthermore, a single nucleotide substitution resulting in premature stop codon in the $t v c$ allele confers resistance to ALV subgroup $C$ [11]. Finally, the tryptophan residue at position 38 (Trp38) of chNHE1 is critical for cell entry by ALV subgroup J [18].

Genetic analyses by our group led to development of cell lines that are resistant to infection by ALV subgroups B and J [19, 20]. Here, we used the same CRISPR/Cas9-based editing approach to develop a new cell line that is resistant to infection by ALV subgroup A. Furthermore, we applied the technology to perform sequential disruption of the $t v a, t v b$, and chNHE1 receptors to develop a cell line that is resistant to all three ALV subgroups.

\section{Materials and methods}

\section{Construction of CRISPR/Cas9 expression vectors}

We constructed all-in-one CRISPR/Cas9 vectors targeting tva, with minor modifications. The CRISPR kit used for constructing multiplex CRISPR/Cas9 vectors was a gift from Takashi Yamamoto (Addgene Kit \#1000000054) [21]. A puromycin resistance gene under the regulation of a thymidine kinase promoter were inserted into CRISPR/ Cas9 vectors by NotI digestion and ligation (Fig. 1a) (New England Biolabs, Ipswich, MA, USA). To insert guide RNA sequences into CRISPR/Cas9 vectors, we synthesized sense and antisense oligonucleotides (Bionics, Seoul, Korea) and carried out annealing using the following thermocycling conditions: $30 \mathrm{~s}$ at $95^{\circ} \mathrm{C}, 2 \mathrm{~min}$ at $72^{\circ} \mathrm{C}, 2 \mathrm{~min}$ at $37^{\circ} \mathrm{C}$, and $2 \mathrm{~min}$ at $25^{\circ} \mathrm{C}$. The oligonucleotides used are listed in Table 1.

\section{Culture of DF-1 chicken fibroblasts}

DF-1 cells were maintained and subpassaged in Dulbecco's minimum essential medium (DMEM; Hyclone, Logan, UT, USA), supplemented with $10 \%$ fetal bovine serum (FBS; Hyclone) and $1 \times$ antibiotic-antimycotic (ABAM; Thermo Fisher-Invitrogen, Carlsbad, CA, USA). DF-1 cells were cultured in an incubator at $37{ }^{\circ} \mathrm{C}$ in an atmosphere of $5 \% \mathrm{CO}_{2}$ at $60 \%-70 \%$ relative humidity.

\section{Transfection and drug selection of DF-1 cells}

CRISPR/Cas9 vectors $(3 \mu \mathrm{g})$ were mixed with Lipofectamine 2000 reagent (Thermo Fisher-Invitrogen) in Opti-MEM (Thermo Fisher-Invitrogen), and the mixture was applied to $5 \times 10^{5} \mathrm{DF}-1$ cells. Then, $6 \mathrm{~h}$ after transfection, transfection mixtures were replaced with DF-1 culture medium. G418 $(300 \mu \mathrm{g} / \mathrm{mL})$ and puromycin $(1 \mu \mathrm{g} /$
$\mathrm{mL}$ ) were added to the culture medium $1 \mathrm{~d}$ after transfection. The complete selection period required up to $7 \mathrm{~d}$.

\section{T7E1 assay}

Genomic DNA was extracted from DF-1 cells after puromycin selection. Genomic regions encompassing the CRISPR/Cas9 target sites were amplified using specific primer sets. PCR analysis of the targeted loci was examined in a total volume of $20 \mu \mathrm{L}$ containing $100 \mathrm{ng}$ genomic DNA, 10× PCR buffer (BioFACT, Daejeon, Korea), $0.4 \mu \mathrm{L}$ dNTPs $(10 \mathrm{mmol} / \mathrm{L}$ each $), 10 \mathrm{pmol}$ of each primer, and $0.5 \mathrm{U}$ Taq polymerase (BioFACT) under the following thermocycling conditions: $2 \mathrm{~min}$ at $95^{\circ} \mathrm{C}$, followed by 35 cycles of $20 \mathrm{~s}$ at $95^{\circ} \mathrm{C}, 40 \mathrm{~s}$ at $65^{\circ} \mathrm{C}$, and $30 \mathrm{~s}$ at $72{ }^{\circ} \mathrm{C}$, and a final $5 \mathrm{~min}$ at $72^{\circ} \mathrm{C}$. Primers are listed in Table 1. The PCR amplicons were re-annealed to form a heteroduplex DNA structure after denaturation. Subsequently, the heteroduplex amplicons were treated with 5 units T7E1 endonuclease (New England Biolabs) for $20 \mathrm{~min}$ at $37^{\circ} \mathrm{C}$ and then analyzed by $1 \%$ agarose gel electrophoresis.

Culture of single DF-1 cells and genomic DNA sequencing After drug selection, the heterozygous DF-1 cells transfected with each CRISPR/Cas9 vectors were diluted with DF-1 culture medium concentration at one cell per $100 \mu \mathrm{L}$, then single DF-1 cells were seeded in individual wells of a 96-well plate. We checked the wells each day after seeding, and when the cells in each well reached confluency, subpassaged the cells into a 48-well plate. These cells were then used for genomic DNA extraction. The genomic regions encompassing the target sites in DF-1 were amplified using specific primer sets (Table 1). PCR analysis of the targeted loci was examined in same condition with T7E1 analysis. And the PCR products were sequenced using the ABI Prism 3730 XL DNA Analyzer (Thermo Fisher-Applied Biosystems, Foster City, CA, USA). The sequences were compared against assembled genomes using NCBI BLAST.

\section{Construction of tva receptor overexpression vector}

The wild type TVA coding sequence was cloned from DF-1 cDNA using wtTVA forward and reverse primers (Table 1), and cloned into piggyBac-CMV-GFP-FRT vector [22] by HindIII and NotI digestion and ligation. The sequences were compared against assembled genomes using NCBI BLAST.

\section{Sequential disruptions of ALV host receptors and genomic DNA sequencing}

Trp38 of chNHE1-modified DF-1 clone (N3Pss\#12) [20] was transfected with the CRISPR/Cas9 vector targeting $t v b$ gene (TVB\#2) [19]. And the DF-1 was seeded in individual wells of a 96-well plate with $100 \mu \mathrm{L}$ DF-1 culture 


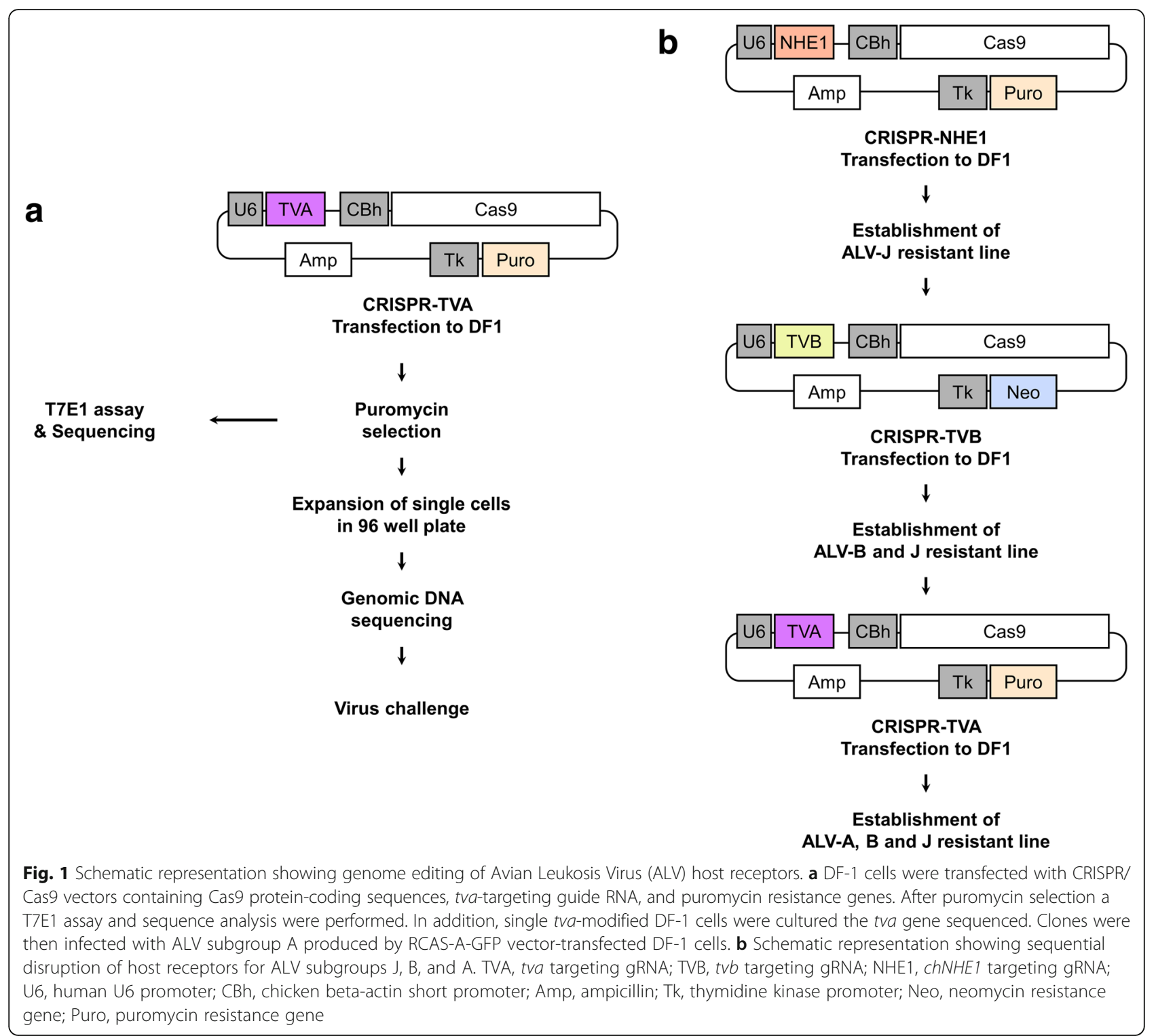

medium. After confirmation of mutations of t $v b$ targeting region in the DF-1 clones by sequencing analysis, the CRISPR/Cas9 vectors targeting tva gene were transfected into the chNHE1 and $t v b$-modified DF-1 clones subsequently and individual DF-1 clones were cultured. And targeting region of $t v a$ gene of the DF-1 clones was analyzed by sequencing analysis (Fig. 1b).

\section{Virus production and infection}

RCAS-A-EGFP DNA, RCAS-B-EGFP DNA and RCAS-J-EGFP DNA ( $5 \mu$ g each) was mixed with Lipofectamine 2000 reagent (Thermo Fisher-Invitrogen) in Opti-MEM (Thermo Fisher-Invitrogen) in separate tubes, and the mixture was applied to $1 \times 10^{6} \mathrm{DF}-1$ cells each. The mixture was replaced with DF- 1 culture medium $6 \mathrm{~h}$ after transfection. One day after transfection, we could observe green fluorescence in DF-1 cells, indicating virus production. Cells were passaged, and the medium was changed $1 \mathrm{~d}$ after passaging. One day later, the medium containing viruses was harvested and frozen at $-70^{\circ} \mathrm{C}$ until further use. For viral infection, the medium containing viruses was thawed to $37^{\circ} \mathrm{C}$ and added to the cultured individual DF-1 clones. Four days post-infection (dpi), DF-1 clones were checked under fluorescence microscopy (TU-80; Nikon, Tokyo, Japan) and analyzed using FACS Calibur software (BD Biosciences, San Jose, CA, USA).

\section{Amino acid sequence analysis}

The amino acid sequences of quail Tva receptor with four chicken Tva isoforms were aligned using the UniProt consortium. 
Table 1 Oligos used in the study

\begin{tabular}{|c|c|}
\hline Oligo ID & Sequence $\left(5^{\prime} \rightarrow 3^{\prime}\right)$ \\
\hline TVA\#1 F & CACCGGCGACGATGGACGGGACGAG \\
\hline TVA\#1 R & AAACCTCGTCCCGTCCATCGTCGCC \\
\hline TVA\#4 F & CACCGCGCTGGAGTGGCTCTGCGAC \\
\hline TVA\#4 R & AAACGTCGCAGAGCCACTCCAGCGC \\
\hline TVA seq $F$ & ACACTGACAGCGAGGCGTGC \\
\hline TVA seq $R$ & ACCTCTCCGCACGACGTTCT \\
\hline wtTVA F & AAGCTTCCGGCGGGCCCGGGGCCGGCATG \\
\hline wtTVA R & GCGGCCGCAGGCAAAAAGAGTGAGGGAATTCC \\
\hline $\operatorname{mutaTVA}(+1) \mathrm{F}$ & $\begin{array}{l}\text { CTGCGACGATGGACGGGACCGAGTGGGGCTGCGG } \\
\text { AGCGAG }\end{array}$ \\
\hline $\operatorname{mutaTVA}(+1) \mathrm{R}$ & $\begin{array}{l}\text { CTCGCTCCGCAGCCCCACTCGGTCCCGTCCATCGTC } \\
\text { GCAG }\end{array}$ \\
\hline $\operatorname{mutaTVA}(-3) \mathrm{F}$ & $\begin{array}{l}\text { CGACTGCGACGATGGACGGGAGTGGGGCTGCGGA } \\
\text { GCGAGC }\end{array}$ \\
\hline $\operatorname{mutaTVA}(-3) \mathrm{R}$ & $\begin{array}{l}\text { GCTCGCTCCGCAGCCCCACTCCCGTCCATCGTCGC } \\
\text { AGTCG }\end{array}$ \\
\hline mutaTVA $(\Delta 1-6) F$ & $\begin{array}{l}\text { ATCCCGACTGCGACGATGGAAAGTGGGGCTGCGG } \\
\text { AGCGAG }\end{array}$ \\
\hline mutaTVA( $\Delta 1-6) R$ & $\begin{array}{l}\text { CTCGCTCCGCAGCCCCACTTTCCATCGTCGCAGTCG } \\
\text { GGAT }\end{array}$ \\
\hline
\end{tabular}

\section{Site directed mutagenesis PCR}

cDNA encoding full length of TVA receptor cloned in pGEM T easy vector (Promega) is used as a template. Mutations were introduced by site-directed mutagenesis PCR, a total volume of $100 \mu \mathrm{L}$ containing $10 \mathrm{ng}$ template vector, $10 \times$ PCR buffer (enzynomics), $0.4 \mu \mathrm{L}$ dNTPs $(10 \mathrm{mmol} / \mathrm{L}$ each), $10 \mathrm{pmol}$ of each primer, and $2.5 \mathrm{U}$ Pfu polymerase (enzynomics) under the following thermocycling conditions: $3 \mathrm{~min}$ at $95^{\circ} \mathrm{C}$, followed by 35 cycles of $30 \mathrm{~s}$ at $95^{\circ} \mathrm{C}, 30 \mathrm{~s}$ at $50{ }^{\circ} \mathrm{C}$, and $4 \mathrm{~min}$ at $72{ }^{\circ} \mathrm{C}$, and a final $15 \mathrm{~min}$ at $72^{\circ} \mathrm{C}$. 3 types of primer sets are used and mutagenic oligonucleotides used are shown in Table 1 . The amplicons were treated with $10 \mathrm{U}$ of DpnI enzyme (New England Biolabs) for $1 \mathrm{~h}$ at $37^{\circ} \mathrm{C}$ and then purified with Wizard SV Gel and PCR Clean-up system (Promega). For phosphorylation, $2 \mu \mathrm{L}$ buffer A (Thermofisher), $10 \mathrm{U}$ T4 polynucleotide kinase (Thermofisher), $2 \mu \mathrm{L}$ $10 \mathrm{mmol} / \mathrm{L}$ ATP (Thermofisher) and $14 \mu \mathrm{L}$ of purified DNA for $1 \mathrm{~h}$ at $37^{\circ} \mathrm{C}$. The phosphorylated PCR products were self-ligated using T4 ligase (Takara), and the mutated TVA sequences were analyzed using the ABI Prism $3730 \mathrm{XL}$ DNA Analyzer (Thermo Fisher-Applied Biosystems, Foster City, CA, USA). Then, the three mutated TVA coding sequences were cloned into piggyBac-CMV-GFP-FRT vector by HindIII and NotI digestion and ligation. The sequences were compared against assembled genomes using NCBI BLAST (http://blast.ncbi.nlm.nih.gov).

\section{Statistical analyses}

Statistical Analysis System (SAS) software (SAS Institute, Cary, NC, USA) was used for the analysis of ALV subgroup A, B and J susceptibility. Treatments were compared using the least-squares method or Duncan's method, and the significance of the main effects was determined using analysis of variance (ANOVA) in the SAS package. A $P$-value $<0.05$ indicated a statistically significant difference.

\section{Results \\ Targeted mutation of the tva gene in chicken DF-1 fibroblasts}

To examine whether disrupting the TVA receptor confers resistance to ALV subgroup A, we used the CRISPR/ Cas 9 system to introduce a targeted mutation in the tva gene of chicken DF-1 fibroblasts. First, we constructed two CRISPR/Cas9 vectors (TVA\#1 and TVA\#4) targeting exon 2 of the tva gene to introduce frameshift mutations into the TVA receptor (Fig. 2a). The CRISPR/Cas9 vectors were then introduced into DF-1 fibroblasts and transfected cells were selected for drug resistance. The results of a T7E1 assay showed that cleaved bands were detected only in DF-1 fibroblasts transfected with each of the CRISPR/Cas9 vectors, indicating that the CRISPR/Cas9 vectors efficiently induced nucleic acid mutations at the targeted locus of the tva gene (Fig. 2b). Sequence analysis of the targeted locus [10] using the TA cloning method confirmed that the DF-1 fibroblasts harbored indel mutations at the targeted regions with $77.8 \%(7 / 9)$ and $66.7 \%(8 / 12)$ efficiency, respectively (Fig. 2c).

\section{Establishment of tva-modified DF-1 clones and subsequent challenge with ALV subgroup A}

To examine susceptibility of $t v a$-modified DF-1 fibroblasts to infection by ALV subgroup A, we generated single clones from DF-1 fibroblasts transfected with TVA\#1 and TVA\#4. We generated eight clones from the two experimental groups, and sequence analysis of the PCR products derived from each clone showed that six of them harbored nucleotide mutations in the tva gene (Additional file 1: Figure S1). Three of these (TVA\#1-6, TVA\#1-8, and TVA\#4-4) harbored frameshift mutations (including deleted- or inserted amino acids) at the targeted locus that generated a premature stop codon in the tva gene. Three other clones (TVA\#1-2, TVA\#1-5, and TVA\#1-7) harbored deletion-, insertion- or substitution- at the targeted locus that did not introduce a frameshift, while two others (TVA\#4-2 and TVA\#4-9) harbored the wild-type (WT) tva allele (Table 2).

Next, we infected each DF-1 clone with ALV subgroup A to examine resistance. Based on expression of green fluorescent protein (GFP) by the marker virus, we found that DF-1 clones harboring nucleic acid mutations in the tva gene (TVA\#1-2, TVA\#1-5, TVA\#1-6, TVA\#1-7, TVA \#1-8, and TVA\#4-4) did not express GFP, 


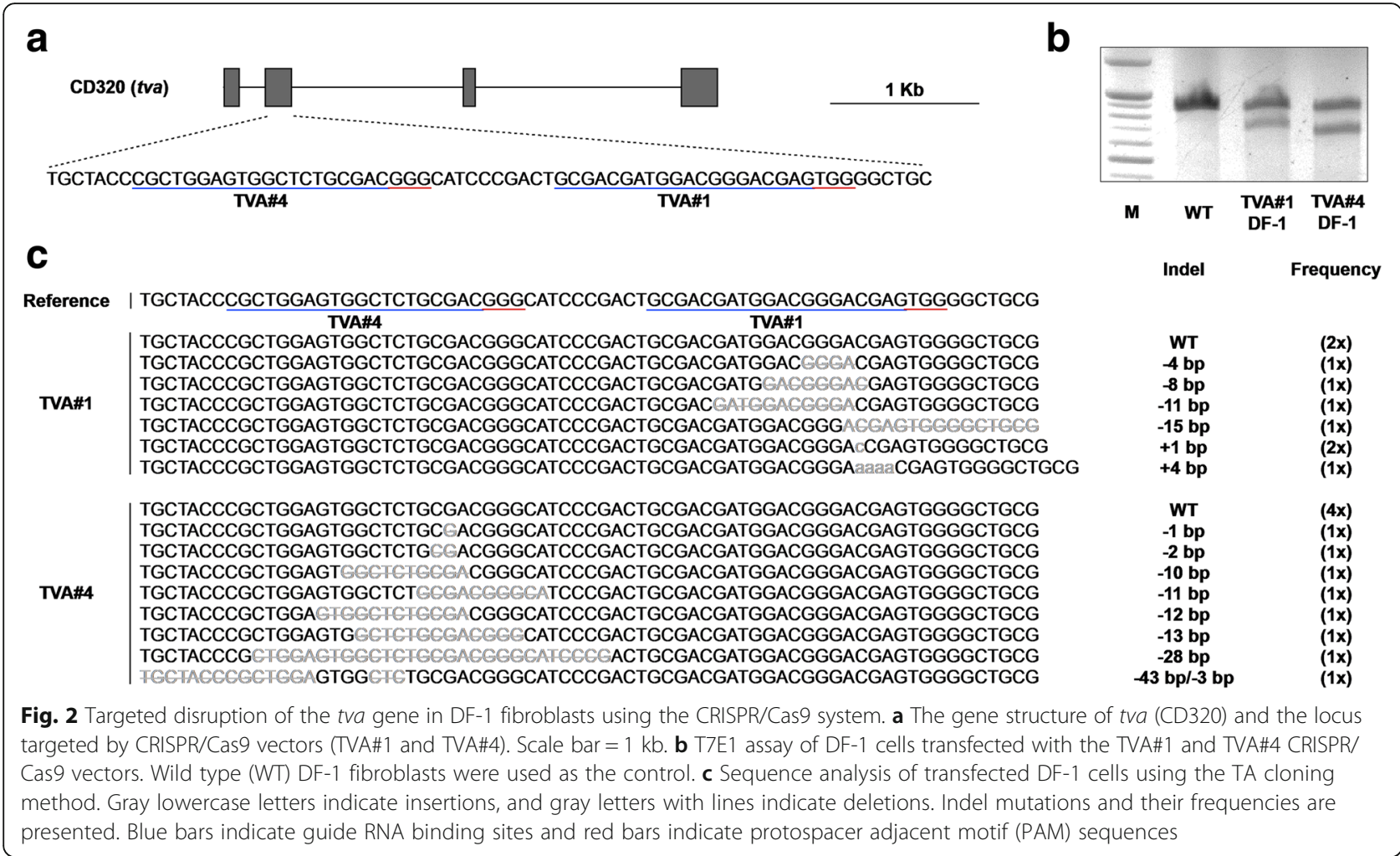

suggesting that ALV subgroup A did not enter these cells; by contrast, virus did enter WT DF-1 control cells (Fig. 3a). Subsequent flow cytometry analysis confirmed that expression of GFP was significantly higher in DF-1 clones harboring the WT tva gene (TVA\#4-2, and TVA\#4-9), indicating that genetic modification of the tva gene confers resistance to ALV subgroup A, regardless of the presence of frameshift mutations (Fig. $3 \mathrm{~b}$ and c). Comparative analysis showed that quail Tva and chicken Tva are highly conserved particularly in the LDLR class A domain (LDLa) (Fig. 3d), and the TVA\#1-2 clone has mutations on the $64^{\text {th }}$ and $65^{\text {th }}$ positions of Tva amino acid, TVA\#1-5 clone has mutation on the $65^{\text {th }}$ position of Tva amino acid and TVA\#1-7 clone has large deletions on from 55 th to 68 th positions of Tva amino acid sequences (Fig. 3e).

\section{Overexpression of the WT TVA receptor by tva-modified DF-1 clones restores susceptibility to infection by ALV subgroup A}

Next, we examined whether overexpressing WT TVA restores susceptibility of $t v a$-modified DF-1 clones to infection by ALV subgroup A. For this, we constructed a piggyBac transposon-based WT TVA overexpression vector (Fig. 4a). Next, we integrated a WT TVA overexpression cassette into tva-modified DF-1 clones (TVA\#1-5, TVA\#1-6, and TVA\#4-4) and WT DF-1 using piggyBac transposase. Analysis of genomic DNA revealed that WT
TVA vector-transfected DF-1 lines contained the WT TVA overexpression cassette (Fig. 4b).

Next, infected DF-1 cell lines with ALV subgroup A and examined their susceptibility to infection. GFP expression was detected in WT TVA-overexpressing cell lines (TVA\#1-5 + TVA, TVA\#1-6 + TVA, and TVA\#4$4+$ TVA) (Fig. 4c). Quantitative analysis using flow cytometry showed that GFP expression by WT TVAoverexpressing groups was significantly higher than that by $t v a$-modified DF-1 clones, although there were differences between the WT TVA-overexpressing groups (Fig. 4d and e).

To further confirm the consequence of mutations on tva for ALV subgroup A susceptibility, we establihsed the DF-1 clones that express modified TVA receptor by piggyBac transposon (Fig. 5a), then examined their susceptibility to infection. As results, GFP expression was not significantly reduced in the modified TVA-overexpressing WT DF-1 cell lines (WT + TVA $(+1)$, WT + TVA $(-3)$, WT + TVA $(\Delta 1-6))$. And, we established the modified TVA receptor-overexpressing DF-1 cell lines from the DF-1 clones that already have a 3-bp pair deletion on tva gene (TVA\#1-5). The results of viral challenge in the cell lines showed that ALV subgroup A susceptibility can not be restored by overexpression of the modified TVA receptors in $t v a$-modified DF- 1 clone (TVA\#1-5 + TVA (+ 1), TVA\#1-5 + TVA (-3), TVA\#1$5+$ TVA $(\Delta 1-6))($ Fig. $5 \mathrm{~b}, \mathrm{c}$ and $\mathrm{d})$. 


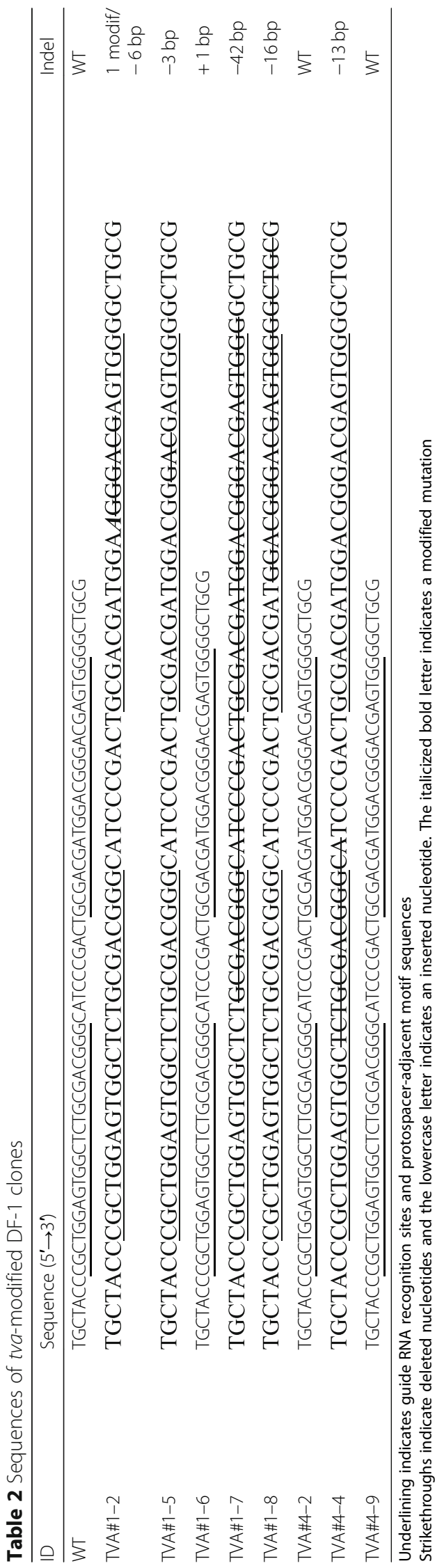




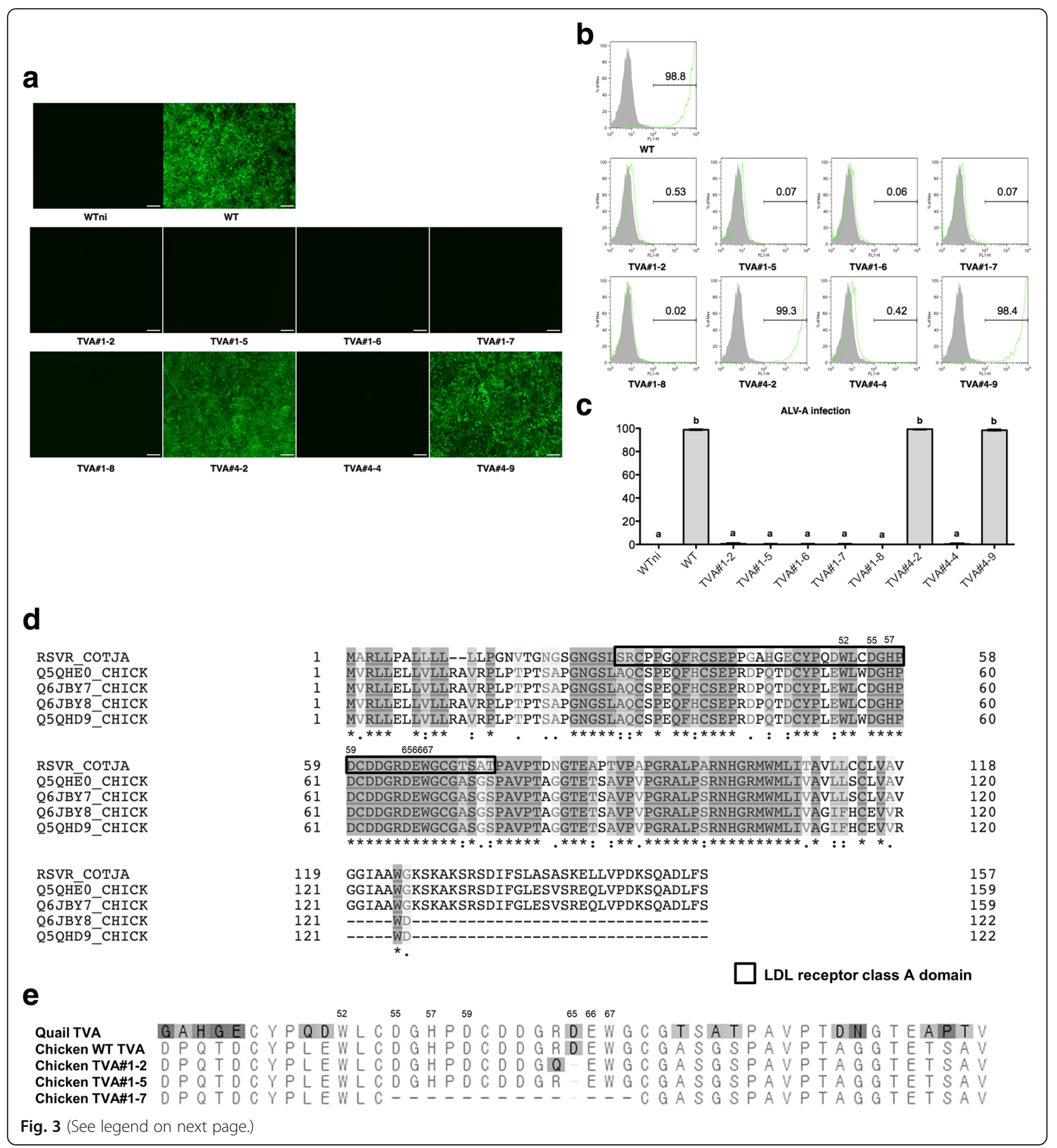


(See figure on previous page.)

Fig. 3 Infection of tva-modified DF-1 clones with avian leukosis virus subgroup A, followed by flow cytometry analysis. a Expression of GFP by virus-infected DF-1 clones. Eight DF-1 clones were examined under a fluorescence microscope. Scale bar $=100 \mu \mathrm{m}$. $\mathbf{b}$, $\mathbf{c}$ Flow cytometry analysis of virus-infected DF-1 clones. Gray peaks indicate the population of wild-type (WT) cells not infected with ALV subgroup A (negative control) and green peaks indicate the population of ALV subgroup A-infected DF-1 clones. The numbers on the histograms represent the mean percentage of cells within that population of triplicate replications. WT DF-1 fibroblasts were used as the control (positive control). c Data represent the mean \pm SEM of triplicate replications. Different letters $(a, b)$ indicate significant differences $(P<0.05)$. $\mathbf{d}$ Multiple-sequence alignment of the four chicken Tva receptor isoforms with the quail Tva receptor. Conserved residues are indicated by the asterisks (identical residues) and dots (similar residues) below the aligned sequences. The quadrangle denotes the viral receptor domain of quail Tva, and the numbers on LDLR class A domain denote the order of quail Tva amino acid sequences. Sequence alignment analysis was conducted using the UniProt consortium. e Comparative analysis of deduced amino acids of tva-modified DF-1 clones. Quail Tva and chicken Tva amino acid are used as references. Tva amino acids of the inframe tva-modified DF-1 clones are presented. TVA\#1-2 clone has mutations on the $64^{\text {th }}$ and the $65^{\text {th }}$ positions of Tva amino acid, TVA\#1-5 clone has mutation on $65^{\text {th }}$ position of Tva amino acid and TVA\#1-7 clone has large deletions on from the $55^{\text {th }}$ to $68^{\text {th }}$ positions of Tva amino acid sequences. Different amino acids are highlighted. The numbers denote the order of quail Tva amino acid sequences

\section{Sequential disruption of host receptors for ALV} subgroups $A, B$ and $J$ and subsequent virus challenge In previous studies, we showed that artificially generating a premature stop codon in the cysteine-rich domain (CRD) of the $t v b$ gene confers resistance to ALV subgroup B [19], and that deletion of chNHE1 Trp38 confers resistance to ALV subgroup J [20]. Therefore, we examined whether sequential editing of the $t v a, t v b$, and chNHE1 genes confers resistance to ALV subgroups A, $\mathrm{B}$, and $\mathrm{J}$ simultaneously.

To induce sequential disruption of host receptors ( $t v a$, $t v b$, and chNHE1), we used a N3Pss\#12 DF-1 clone in which chNHE1 Trp38 was deleted [20]. Sequential transfection of CRISPR/Cas9 vectors targeting the $t v b$ and a

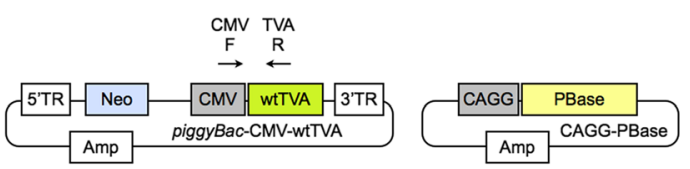

b

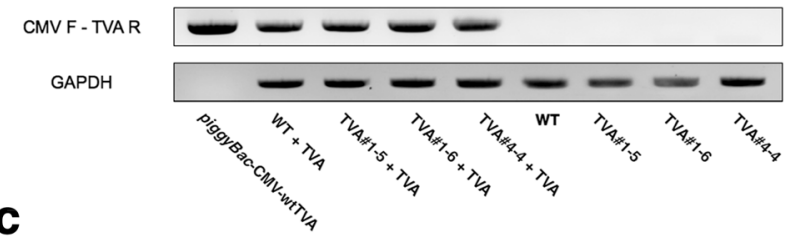

d
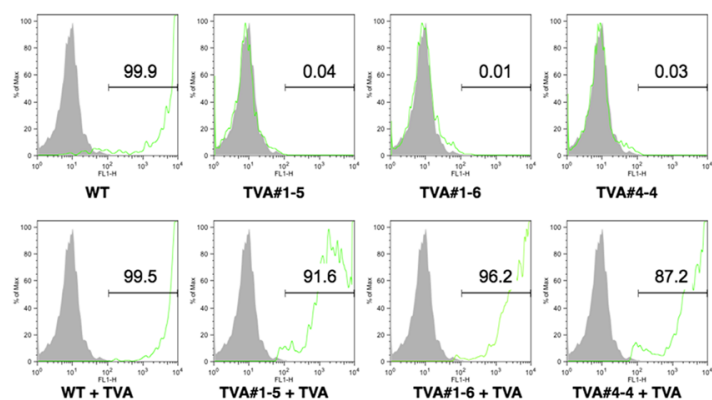

TVA:11-5

TVA\#1-6
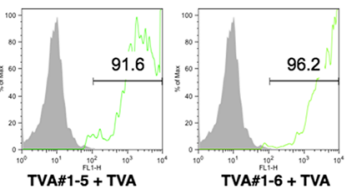

TVA\#4

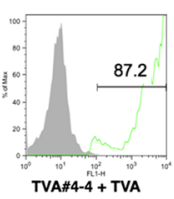

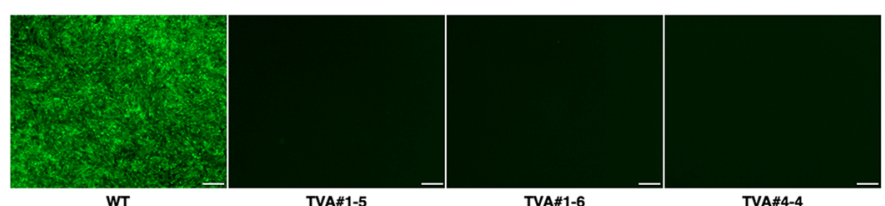

e

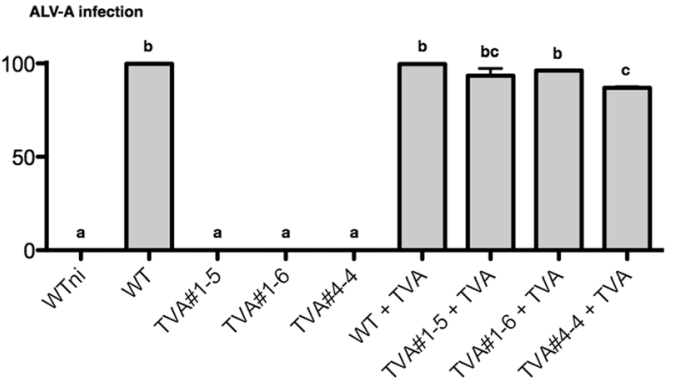

Fig. 4 Overexpression of the wild-type (WT) TVA receptor restores susceptibility of tva-modified DF-1 clones to infection by ALV subgroup A. a Schematic representation showing the wild-type TVA receptor (WT TVA) overexpression cassette. 5'-TR: piggyBac 5'-terminal repeat; Neo: neomycin resistant gene; CMV: cytomegalovirus promoter; WT TVA: WT TVA coding sequence; 3'-TR: piggyBac 3'-terminal repeat; Amp: ampicillin resistant gene; CAGG: cytomegalovirus (CMV) enhancer fused to the chicken beta-actin promoter; PBase: piggyBac transposase coding sequence. $\mathbf{b}$ Analysis of genomic DNA to confirm integration of the WT TVA overexpression cassette under specific PCR conditions. GAPDH was used as a control. c Expression of GFP by virus-infected DF-1 clones. Three DF-1 clones (TVA\#1-5, TVA\#1-6, and TVA\#4-4) and WT DF-1 cells were transfected with the WT TVA overexpression cassette and then infected with ALV subgroup A. GFP expression by each group was examined under a fluorescence microscope. Scale bar $=100 \mu \mathrm{m}$. d, e Flow cytometry analysis of virus-infected DF-1 clones. Gray peaks indicate the population of WT cells not infected with ALV subgroup A (negative control) and green peaks indicate the population of DF-1 clones infected with ALV subgroup A. The numbers on the histograms represent the mean percentage of cells within that population of triplicate replications. WT DF-1 fibroblasts were used as the control (positive control). Different letters ( $a$, b and c) indicate significant differences $(P<0.05)$ 
a

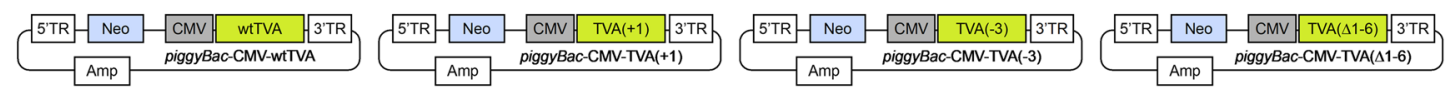

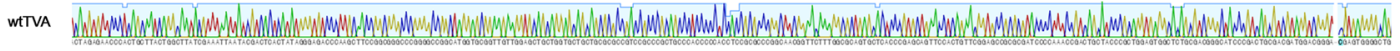

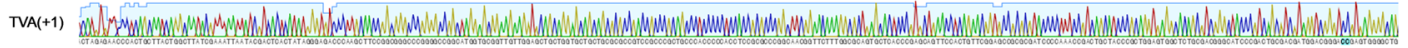

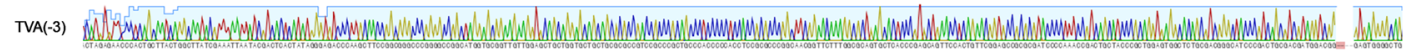

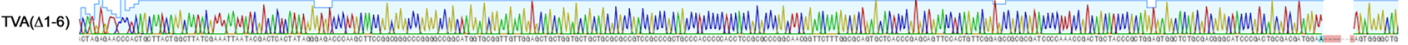

b
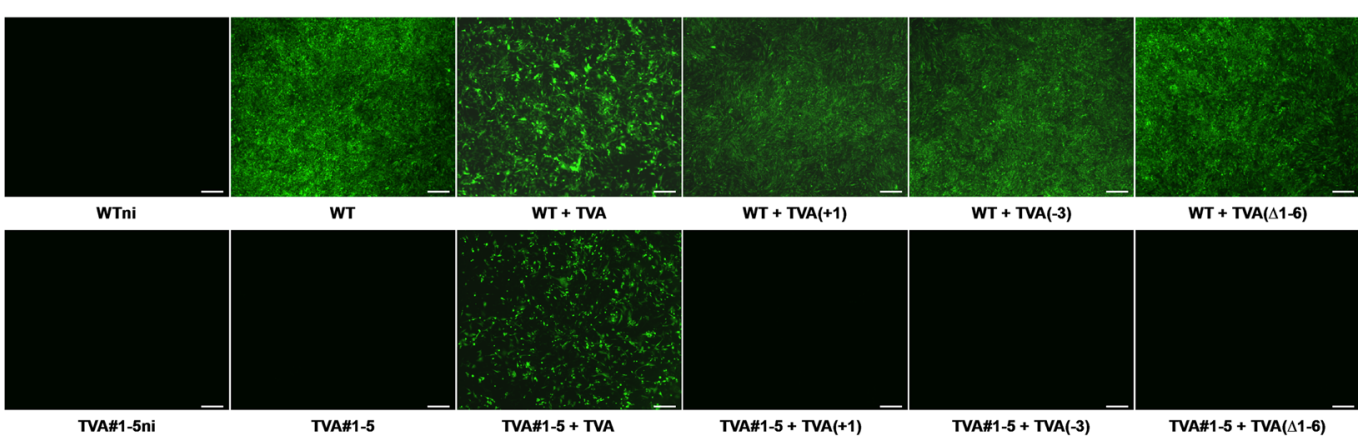

C
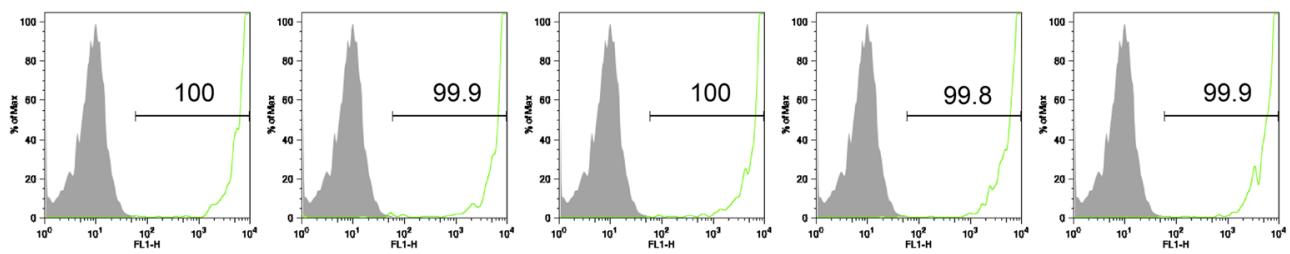

WT

WT + TVA

WT + TVA(+1)

WT + TVA(-3)

WT + TVA $(\Delta 1-6)$
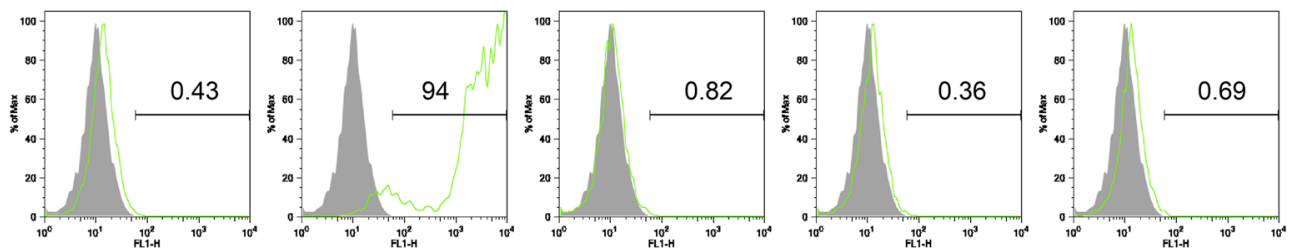

TVA\#1-5

TVA\#1-5 + TVA

TVA\#1-5 + TVA(+1)

TVA\#1-5 + TVA(-3)

TVA\#1-5 + TVA( $\triangle 1-6)$

d

ALV-A infection
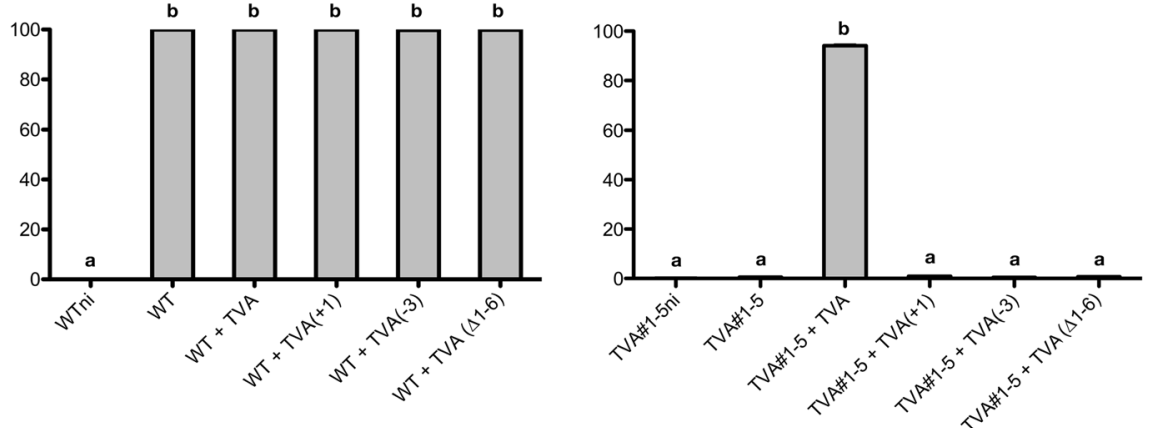

Fig. 5 (See legend on next page.) 


\section{(See figure on previous page.)}

Fig. 5 a Schematic representation showing the wild-type TVA receptor (WT TVA) and modified TVA receptor (TVA $(+1)$, TVA $(-3)$ and TVA( $\triangle 1-6)$ ) overexpression cassette and sequencing results of the constructed vector. CMV promoter and TVA CDS sequences are described, and modified nucleic acids are highlighted. 5'-TR: piggyBac 5'-terminal repeat; Neo: neomycin resistant gene; CMV: cytomegalovirus promoter; 3'-TR: piggyBac 3'terminal repeat; Amp: ampicillin resistant gene; CAGG: cytomegalovirus (CMV) enhancer fused to the chicken beta-actin promoter; PBase: piggyBac transposase coding sequence. $\mathbf{b}$ Expression of GFP by virus-infected DF-1 clones. WT TVA or modified TVA receptors overexpessing WT DF-1 cell lines $(\mathrm{WT}, \mathrm{WT}+\mathrm{TVA}, \mathrm{WT}+\mathrm{TVA}(+1)$, WT + TVA(-3) and WT + TVA( $\triangle 1-6)$ ) and tva-modified DF-1 clones (3 bp deletion on tvb) (TVA\#1-5, TVA\#1-5 + TVA,

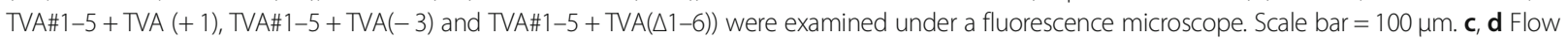
cytometry analysis of virus-infected DF-1 clones. Gray peaks indicate the population of the DF-1 not infected with ALV subgroup A (negative control; WTni and TVA\#1-5ni) and green peaks indicate the population of ALV subgroup A-infected DF-1 clones. The numbers on the histograms represent the mean percentage of cells within that population of triplicate replications. WT DF-1 fibroblasts were used as the control (positive control). $\mathbf{d}$ Data represent the mean \pm SEM of triplicate replications. Different letters $(a, b)$ indicate significant differences $(P<0.05)$

tva genes into the chNHE1-modified clone generated DF-1 clones harboring nucleic acid mutations in the $t v a$, $t v b$, and $c h N H E 1$ genes. First, we confirmed that the N3Pss\#12 DF-1 clone harbored a mutation in chNHE1 by sequencing a PCR product amplified from the target region (Additional file 1: Figure S2A) [20]. Next, we confirmed that the N3Pss\#12+TVB\#2 clone harbored an 8 -bp deletion in the $t v b$ gene that generated a premature stop codon (Additional file 1: Figure S2B). Finally, we confirmed that the three DF-1 clones (N3Pss\#12+ TVB\#2 + TVA\#1-6, N3Pss\#12 + TVB\#2 + TVA\#1-8, and N3Pss\#12 + TVB\#2 + TVA\#4-8) established by sequential transfection of CRISPR/Cas9 vectors targeting $t v b$ and tva harbored the same mutations in $t v b$ as the N3Pss\#12 + TVB\#2 clones, as well as the same deletion mutations in the tva gene (Additional file 1: Figure S2C) and the mutations in the chNHE1 (Table 3).

\section{Challenge of chNHE1, tvb, and tva-modified DF-1 clones with ALV subgroup A, B and J}

To identify the susceptibility of chNHE1-, tvb-, and tvamodified DF-1 clones to infection by ALV, we infected each clone with GFP-expressing marker viruses belonging to ALV subgroups A, B, and J, respectively. GFP expression was detected when the chNHE1-modified clone (N3Pss\#12) was infected with either ALV subgroup A and $B$, but not upon infection with ALV subgroup J. In addition, GFP expression was detected only when chNHE1/tvb-modified clones (N3Pss\#12+TVB\#2) were infected with ALV subgroup A, but not upon infection with ALV subgroups B and J. No GFP expression was detected in chNHE1/tvb/tva-modified DF-1 clones (N3Pss\#12 + TVB\#2 + TVA\#1-6, N3Pss\#12 + TVB\#2 + TVA\#1-8, and N3Pss\#12 + TVB\#2 + TVA\#4-8) after infection by ALV subgroups A, B, and J (Fig. 6a). Subsequent flow cytometry analysis showed marked GFP expression by DF-1 clones harboring WT host genes (Fig. 6b and c).

\section{Discussion}

Successfully controlling infectious diseases is one of the most important challenges facing the poultry industry.
Enhancing economic traits using programmable genome editing technologies such as CRISPR/Cas9 has merit in terms of reducing the time required to generate a desired genotype in livestock species [23]. In particular, programmable genome editing technology can be applied directly to develop resistance to specific viral diseases such as ALV, for which the host receptors have been identified. Here, we used genome editing technology to develop cell lines that are resistance to infection by multiple ALVs; this step will precede development of genome-edited chicken lines.

As reported previously, ALV subgroup A enters host cells via the TVA receptor [7]. Deletion of introns from the tva gene disrupts mRNA splicing and/or induces frameshift mutations in exon 2, thereby altering host cell susceptibility to infection by ALV subgroup A [13-15]. Here, we induced a frameshift mutation into the tva gene rather than modifying the intron region to confer resistance to ALV subgroup A. The two CRISPR/Cas9 vector constructs used induced nucleic acid mutations within the targeted regions, and the pattern of mutations correspond to those observed in our previous studies $[19,20]$. The results indicated that the CRISPR/Cas9-based gene editing system is an efficient tool for targeting specific regions of the chicken genome. However, we were unable to induce the exact mutation in the tva gene that is reported to confer resistance [13]. The experiments could be improved by using HDR-based approaches as well as by evaluating off-target mutations.

By contrast, the results of virus challenge experiments showed that nucleotide mutations in the tva gene that result in frameshift mutations are crucial for ALV subgroup A entry. The TVA receptor belongs to LDLR family; indeed, the LDLa plays an important role in receptor function [24]. Exon 2 of the tva gene contains the LDLa; therefore, we suggest that frameshift mutations causing disruption of the LDLa domain would lead to misfolding of the TVA protein. Furthermore, the results of virus challenge experiments in Asp67-deleted (TVA\#1-5) and Asp67- and Glu68-deleted (TVA\#1-2) DF-1 clones (these mutations do not cause a frameshift) also conferred resistance to ALV subgroup A. These results 


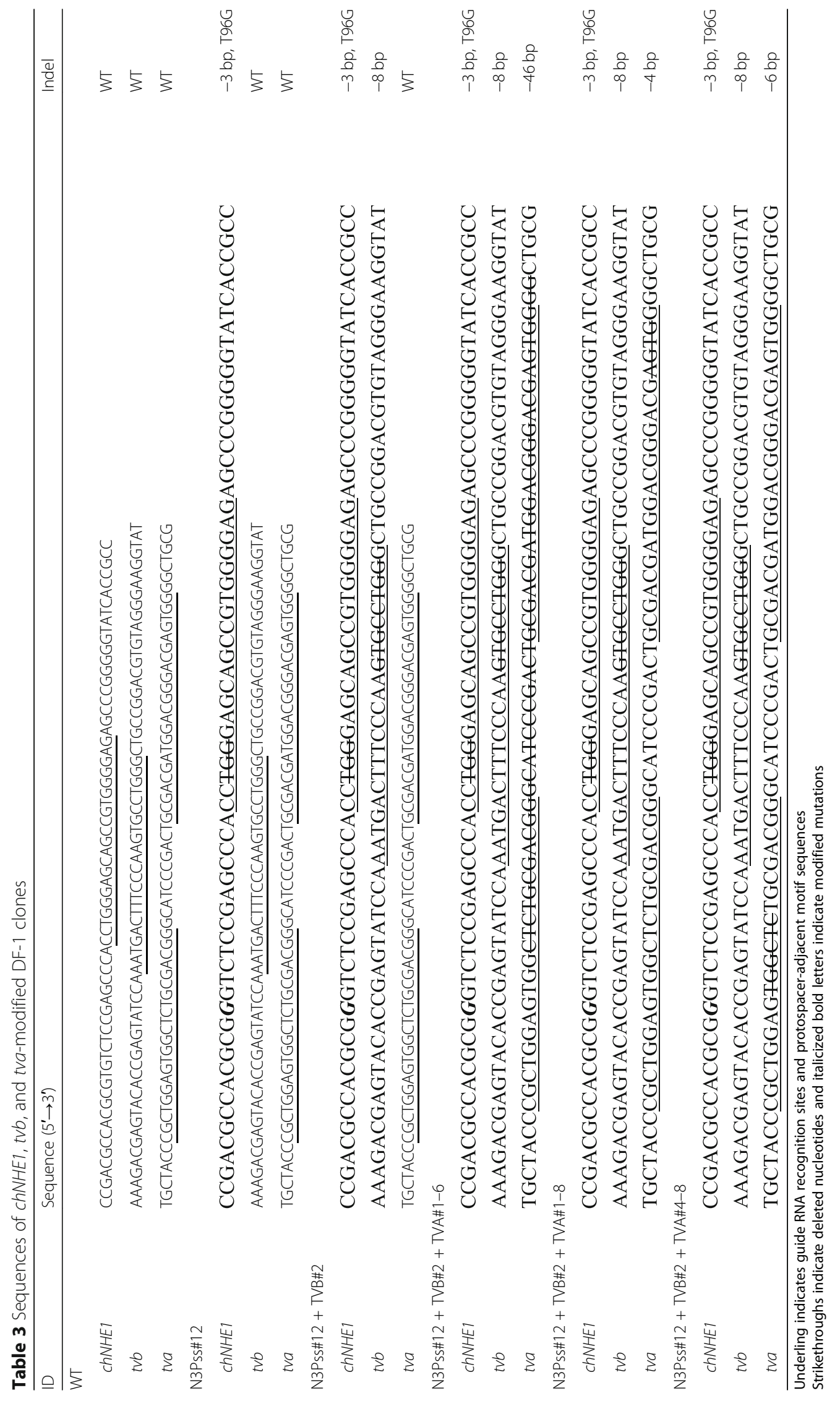




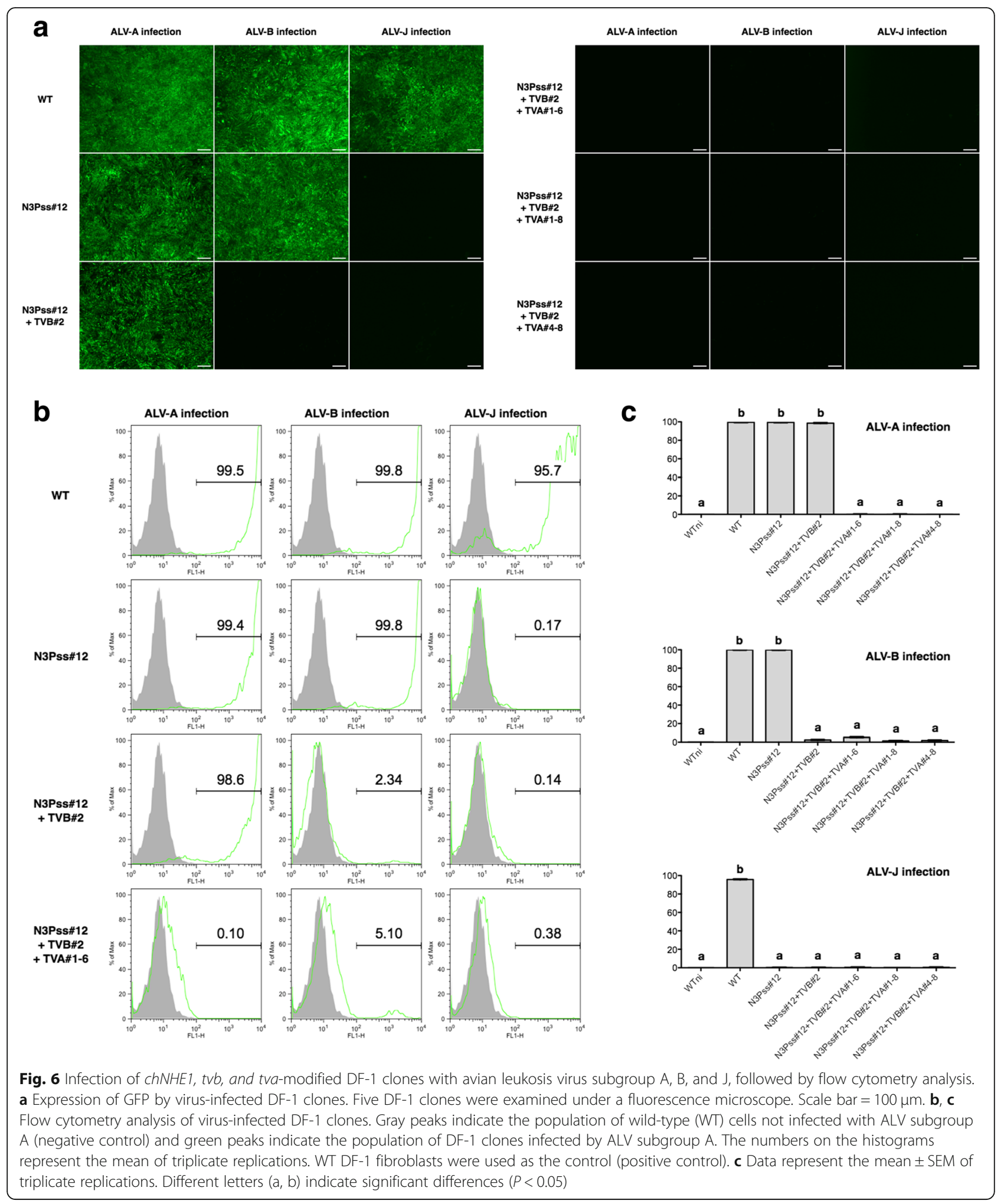

correspond with those published in previous studies examining ALV-A susceptibility linked to the TVA receptor [25]; two acidic amino acids in the carboxy-terminal portion of TVA receptor and the frameshift mutations are indispensable for infection by ALV subgroup A. Previous studies show that Trp52, Asp55, His57, Asp59, Asp65, and Glu66 of the quail TVA protein are calciumcoordinating sites for the TVA LDL-A module and are 
required for proper protein folding. Furthermore, His57 and Trp67 of the quail TVA protein act as viral contact residues and are involved directly in binding to EnvA [26, 27]. Protein structure analysis revealed that the LDLR region harboring these residues is highly conserved in different chicken TVA receptor isoforms. These results suggest that the in-frame tva-modified DF-1 clones have impaired function due to improper protein folding caused by a lack of calcium binding residues. Also, they do not bind to EnvA due to lack of viral contact residues. Moreover, we showed that overexpression of WT TVA restores susceptibility to infection by ALV subgroup A viruses. However, differences in susceptibility were noted between cell lines containing the WT TVA overexpression cassette, possibly due to the differences in piggyBac integration sites and copy number of transgene [22]. Meanwhile, modified TVA receptors could not restore susceptibility to infection by ALV subgroup A viruses in tva-modified DF-1 clone. Taken together, the results indicate that the TVA receptor is necessary for entry of ALV subgroup A viruses and could therefore be targeted to alter the immune characteristics of poultry flocks.

We also found that sequential disruption of ALV host receptors chNHE1, TVB, and TVA confers resistance to ALV subgroups A, B and J, respectively. We examined this by modifying the host receptor genes in chicken DF-1 fibroblasts by sequential introduction of CRISPR/Cas9 gene editing vectors. The results demonstrated that altering multiple genes can be achieved; this technique can then be used to develop multiple gene knock-out cell lines. Subsequent virus infection experiments showed that disruptions of the $t v a-$, $t v b$-, and $c h N H E 1$-encoded receptors conferred simultaneous resistance to ALV subgroups A, B and $\mathrm{J}$. As expected, the results also confirmed that ALV subgroups A, B and J do not share the same receptors for entry into cells, despite the close sequence similarity between ALV subgroups A and B [28]. By understanding interactions between ALVs and host receptors at the genetic level we may be able to predict likely host receptors for any emerging subgroup of ALV.

\section{Conclusion}

We conducted multiplex genome editing of chicken DF-1 fibroblasts and showed that sequential disruption of host receptors for ALV subgroups A, B, and J confers resistance. To gain further insight into virus-host interactions, as well as into virus evolution, we will analyze the susceptibility of these cells to other virus subgroups (such as ALV subgroup C and the recently reported subgroup K). As a next step, we will apply the genome editing strategy to germline competent cells, including primordial germ cells or spermatogonial stem cells [29, 30], to generate novel multi-disease resistant chicken lines as an approach to controlling major avian diseases.
Indeed, NHE1-null fibroblasts show impaired homeostasis, manifested by reduced adhesion, loss of polarity, and reduced motility and chemotaxis [31]. However, chicken cell lines naturally resistant to ALV subgroups A and B are available (e.g., inbred chicken line $\mathrm{C}$ and line $7_{2}$ ), as are avian species harboring mutations in the Trp38 of the NHE1 receptor $[13,18]$. These results suggest that the ALV host receptors may be not be critical, or that other receptors compensate for a specific function of each receptor. To evaluate the function of each receptor, physiological and molecular analyses of immune responses and cell homeostasis in receptor gene-modified cells or birds are needed.

\section{Additional file}

Additional file 1: Figure S1. Sequences of the targeted regions within tva-modified DF-1 clones. Red arrows indicate TVA\#1 and TVA\#4 targeting regions. A reference sequence is shown. Figure S2. Sequences of targeted regions within (a) ChNHE1, (b) chNHE1 and tvb, and (c) chNHE1, tvb, and tva-modified DF-1 clones. Red arrows indicate the (a) NHE1\#3, (b) TVB\#2, and (c) TVA\#1 and TVA\#4 targeting regions. Reference sequences are shown. (DOCX $848 \mathrm{~kb}$ )

\section{Abbreviations}

ABAM: Antibiotic-antimycotic; ALV: Avian leukosis virus; ANOVA: Analysis of variance; chNHE1: $\mathrm{Na}^{+} / \mathrm{H}^{+}$exchange 1; CRD: Cysteine-rich domain; CRISPR/ Cas9: Clustered regularly interspaced short palindromic repeats (CRISPR)/ CRISPR-associated protein 9; DMEM: Dulbecco's minimum essential medium; FBS: Fetal bovine serum; GFP: Green fluorescent protein; LDLa: Low-density lipoprotein receptors (LDLR) class A domain; PGCs: Primordial germ cells; SAS: Statistical analysis system; SSCs: Spermatogonial stem cells; TNFR: Tumor necrosis factor receptor; Trp38: Tryptophan residue at position 38; WT: Wild type; wtTVA: Wild type TVA receptor

\section{Acknowledgements}

Not applicable.

\section{Funding}

This work was supported by This work was supported by the National Research Foundation of Korea grant funded by the Korea government (MSIP) (NRF2015R1A3A2033826), and "Cooperative Research Program for Agriculture Science and Technology Development (Project No. PJ0131642018)" Rural Development Administration, Republic of Korea. Also this work was supproted by the Royal Society International Professorships (No.IC160046) and Biotechnology and BiologicalSciences Research Council (BBSRC) Partnership award (BB/M027481/1), UK

\section{Availability of data and materials}

The datasets during and/or analyzed during the current study available from the corresponding authors on reasonable request.

\section{Authors' contributions}

JYH participated in study design and coordination. HJL and KJP participated in the design of the study, carried out the experiments, statistical analysis and wrote the first draft of the manuscript. KYL carried out and analyzed the experiments. $Y Y$ and VN participated in writing the final versions of the manuscript. All authors have read and approved the final manuscript.

\section{Ethics approval}

The care and experimental use of chickens were approved by the Institute of Laboratory Animal Resources, Seoul National University. 


\section{Competing interests}

The authors declare that they have no competing interests.

\section{Author details}

'Department of Agricultural Biotechnology, College of Agriculture and Life Sciences, and Research Institute of Agriculture and Life Sciences, Seoul National University, Seoul 08826, Korea. ${ }^{2}$ The Pirbright Institute, Woking, Surrey GU24 ONF, UK.

Received: 27 October 2018 Accepted: 10 February 2019

Published online: 02 April 2019

\section{References}

1. Justice J, Beemon KL. Avian retroviral replication. Curr Opin Virol. 2013;3: $664-9$.

2. Dong X, Zhao P, Xu B, Fan J, Meng F, Sun P, et al. Avian leukosis virus in indigenous chicken breeds, China. Emerg Microbes Infect. 2015;4:e76.

3. Lin W, Li X, Dai Z, Zhang X, Chang S, Zhao P, et al. Molecular epidemiology of J-subgroup avian leukosis virus isolated from meat-type chickens in southern China between 2013 and 2014. Arch Virol. 2016;161:3039-46.

4. Pan W, Gao Y, Sun F, Qin L, Liu Z, Yun B, et al. Novel sequences of subgroup $\mathrm{J}$ avian leukosis viruses associated with hemangioma in Chinese layer hens. Virol J. 2011;8:552.

5. Wang P, Lin L, Li H, Yang Y, Huang T, Wei P. Diversity and evolution analysis of glycoprotein GP85 from avian leukosis virus subgroup J isolates from chickens of different genetic backgrounds during 1989-2016: coexistence of five extremely different clusters. Arch Virol. 2018;163:377-89.

6. Payne LN, Howes K, Gillespie AM, Smith LM. Host range of Rous sarcoma virus pseudotype RSV(HPRS-103) in 12 avian species: support for a new avian retrovirus envelope subgroup, designated J. J Gen Virol. 1992;73(Pt 11):2995-7.

7. Bates $P$, Young JA, Varmus HE. A receptor for subgroup A Rous sarcoma virus is related to the low density lipoprotein receptor. Cell. 1993;74:1043-51.

8. Brojatsch J, Naughton J, Rolls MM, Zingler K, Young JA. CAR1, a TNFRrelated protein, is a cellular receptor for cytopathic avian leukosis-sarcoma viruses and mediates apoptosis. Cell. 1996:87:845-55.

9. Adkins HB, Brojatsch J, Naughton J, Rolls MM, Pesola JM, Young JA. Identification of a cellular receptor for subgroup $\mathrm{E}$ avian leukosis virus. Proc Natl Acad Sci U S A. 1997;94:11617-22.

10. Adkins HB, Blacklow SC, Young JA. Two functionally distinct forms of a retroviral receptor explain the nonreciprocal receptor interference among subgroups B, D, and E avian leukosis viruses. J Virol. 2001;75:3520-6.

11. Elleder D, Stepanets V, Melder DC, Senigl F, Geryk J, Pajer P, et al. The receptor for the subgroup $C$ avian sarcoma and leukosis viruses, Tvc, is related to mammalian butyrophilins, members of the immunoglobulin superfamily. J Virol. 2005;79:10408-19.

12. Chai $\mathrm{N}$, Bates $\mathrm{P}$. Na+/H+ exchanger type 1 is a receptor for pathogenic subgroup J avian leukosis virus. Proc Natl Acad Sci U S A. 2006;103:5531-6.

13. Elleder D, Melder DC, Trejbalova K, Svoboda J, Federspiel MJ. Two different molecular defects in the Tva receptor gene explain the resistance of two tvar lines of chickens to infection by subgroup A avian sarcoma and leukosis viruses. J Virol. 2004:78:13489-500

14. Chen W, Liu Y, Li H, Chang S, Shu D, Zhang H, et al. Intronic deletions of tva receptor gene decrease the susceptibility to infection by avian sarcoma and leukosis virus subgroup a. Sci Rep. 2015;5:9900.

15. Reinisova M, Plachy J, Trejbalova K, Senigl F, Kucerova D, Geryk J, et al. Intronic deletions that disrupt mRNA splicing of the tva receptor gene result in decreased susceptibility to infection by avian sarcoma and leukosis virus subgroup a. J Virol. 2012;86:2021-30.

16. Klucking $S$, Adkins $H B$, Young JA. Resistance to infection by subgroups $B, D$, and $E$ avian sarcoma and leukosis viruses is explained by a premature stop codon within a resistance allele of the tvb receptor gene. J Virol. 2002;76: 7918-21.

17. Reinisova $M$, Senigl F, Yin X, Plachy J, Geryk J, Elleder D, et al. A singleamino-acid substitution in the TVbS1 receptor results in decreased susceptibility to infection by avian sarcoma and leukosis virus subgroups B and $D$ and resistance to infection by subgroup $E$ in vitro and in vivo. J Virol. 2008:82:2097-105.

18. Kucerova D, Plachy J, Reinisova M, Senigl F, Trejbalova K, Geryk J, et al. Nonconserved tryptophan 38 of the cell surface receptor for subgroup
$\mathrm{J}$ avian leukosis virus discriminates sensitive from resistant avian species. J Virol. 2013:87:8399-407.

19. Lee HJ, Lee KY, Park YH, Choi HJ, Yao Y, Nair V, et al. Acquisition of resistance to avian leukosis virus subgroup $B$ through mutations on tvb cysteine-rich domains in DF-1 chicken fibroblasts. Vet Res. 2017:48:48.

20. Lee HJ, Lee KY, Jung KM, Park KJ, Lee KO, Suh JY, et al. Precise gene editing of chicken $\mathrm{Na}+/ \mathrm{H}+$ exchange type 1 (chNHE1) confers resistance to avian leukosis virus subgroup J (ALV-J). Dev Comp Immunol. 2017;77:340-9.

21. Sakuma T, Nishikawa A, Kume S, Chayama K, Yamamoto T. Multiplex genome engineering in human cells using all-in-one CRISPR/Cas9 vector system. Sci Rep. 2014:4:5400.

22. Lee HJ, Lee HC, Kim YM, Hwang YS, Park YH, Park TS, et al. Site-specific recombination in the chicken genome using Flipase recombinase-mediated cassette exchange. FASEB J. 2016;30:555-63.

23. Lee HJ, Kim YM, Ono T, Han JY. Genome modification technologies and their applications in avian species. Int J Mol Sci. 2017;18:2245.

24. Yamamoto T, Davis CG, Brown MS, Schneider WJ, Casey ML, Goldstein JL, et al. The human LDL receptor: a cysteine-rich protein with multiple Alu sequences in its mRNA. Cell. 1984:39:27-38.

25. Zingler K, Belanger C, Peters R, Agard E, Young JA. Identification and characterization of the viral interaction determinant of the subgroup $A$ avian leukosis virus receptor. J Virol. 1995:69:4261-6.

26. Wang QY, Dolmer K, Huang W, Gettins PG, Rong L. Role of calcium in protein folding and function of Tva, the receptor of subgroup $A$ avian sarcoma and leukosis virus. J Virol. 2001;75:2051-8.

27. Wang OY, Huang W, Dolmer K, Gettins PG, Rong L. Solution structure of the viral receptor domain of Tva and its implications in viral entry. J Virol. 2002; 76:2848-56

28. Li X, Lin W, Chang S, Zhao P, Zhang X, Liu Y, et al. Isolation, identification and evolution analysis of a novel subgroup of avian leukosis virus isolated from a local Chinese yellow broiler in South China. Arch Virol. 2016;161: $2717-25$.

29. Choi JW, Kim S, Kim TM, Kim YM, Seo HW, Park TS, et al. Basic fibroblast growth factor activates MEK/ERK cell signaling pathway and stimulates the proliferation of chicken primordial germ cells. PLoS One. 2010:5:e12968.

30. Kim YM, Park JS, Yoon JW, Choi HJ, Park KJ, Ono T, et al. Production of germline chimeric quails following spermatogonial cell transplantation in busulfan-treated testis. Asian J Androl. 2018;20:414-6.

31. Denker SP, Barber DL. Cell migration requires both ion translocation and cytoskeletal anchoring by the Na-H exchanger NHE1. J Cell Biol. 2002;159: 1087-96.

Ready to submit your research? Choose BMC and benefit from:

- fast, convenient online submission

- thorough peer review by experienced researchers in your field

- rapid publication on acceptance

- support for research data, including large and complex data types

- gold Open Access which fosters wider collaboration and increased citations

- maximum visibility for your research: over $100 \mathrm{M}$ website views per year

At $\mathrm{BMC}$, research is always in progress.

Learn more biomedcentral.com/submission 